\title{
Rupture prediction of intracranial aneurysms: a nationwide matched case-control study of hemodynamics at the time of diagnosis
}

\author{
Torbjørn Øygard Skodvin, MD, ${ }^{1,2}$ Øyvind Evju, MSc, PhD, ${ }^{3}$ Christian A. Helland, MD, PhD,, 45 and \\ Jørgen Gjernes Isaksen, MD, PhD ${ }^{1,2}$
}

\begin{abstract}
${ }^{1}$ Faculty of Health, UiT The Arctic University of Norway, Tromsø; ' 2 Department of Neurosurgery, University Hospital of Northern Norway, Troms $\varnothing ;{ }^{3}$ Simula Research Laboratory, Oslo; ${ }^{4}$ Department of Neurosurgery, Haukeland University Hospital, Bergen; and ${ }^{5}$ Department of Clinical Medicine, University of Bergen, Norway
\end{abstract}

\begin{abstract}
OBJECTIVE Hemodynamic rupture predictors in intracranial aneurysms (IAs) must be investigated in prerupture aneurysms, because rupture might significantly alter aneurysm hemodynamics. Both clinical and aneurysmal factors influence hemodynamics, possibly confounding results in unmatched patient materials. The authors aimed to identify hemodynamic variables at the time of diagnosis that could be associated with IA rupture.
\end{abstract}

METHODS A nationwide matched case-control study of IA hemodynamics at the time of diagnosis was performed. Twelve IAs that later ruptured were matched 1:2 with control aneurysms that remained unruptured during a median follow-up time of 4.5 years (interquartile range 3.7-8.2 years). Cases and controls were matched by aneurysm location and size, and patient sex and age. Hemodynamic parameters were obtained from computational fluid dynamics simulations. RESULTS The low shear area (LSA) was significantly higher in cases than in controls in univariate analysis $(p=0.041)$. Minimum logarithmic wall shear stress, averaged logarithmic wall shear stress, pressure loss coefficient, and inflow concentration index showed a tendency to be associated with later rupture $(p=0.09,0.14,0.15$, and 0.18 , respectively). The LSA remained statistically significant in multivariable analysis $(p=0.030)$.

CONCLUSIONS Hemodynamics at the time of diagnosis are different in aneurysms that later rupture than in those that remain unruptured. Increased LSA might be an early predictor of rupture.

https://thejns.org/doi/abs/10.3171/2017.5.JNS17195

KEY WORDS intracranial aneurysm; hemodynamics; computational fluid dynamics; subarachnoid hemorrhage; rupture; vascular disorders

$\mathrm{S}$ UBARACHNOID hemorrhage (SAH) is an important cause of premature death and socioeconomic cost. ${ }^{13,25}$ Saccular intracranial aneurysms (IAs) are the cause of $85 \%$ of SAH. ${ }^{30}$ These IAs are found in approximately $2 \%-3.5 \%$ of the adult population, but the individual rupture risk is uncertain. ${ }^{21,31}$ Prophylactic surgical or endovascular treatment of aneurysms minimizes the chances of future rupture, but involves considerable risk. Wider use and availability of radiological imaging of the brain has increased the rate of incidentally discovered IAs. Highgrade guidelines for the management of unruptured IAs are lacking, ${ }^{27}$ warranting improved methods for rupture risk assessment. Evaluation of aneurysm hemodynamics can possibly aid treatment decisions, because studies show that the hemodynamics of ruptured aneurysms are different from those for unruptured lesions. ${ }^{3}$ Frequently studied hemodynamic parameters include derivatives of wall shear stress (WSS), such as oscillatory shear index (OSI) and low shear area (LSA), ${ }^{5}$ and parameters related to the flow phenotype, such as pressure loss coefficient (PLc), inflow concentration index (ICI), and viscous dissipation ratio (VDR). ${ }^{4,28}$

ABBREVIATIONS ACOA = anterior communicating artery; CFD = computational fluid dynamics; $I \mathrm{~A}=$ intracranial aneurysm; ICA = internal carotid artery; ICI = inflow concentration index; IQR = interquartile range; $L S A=$ Iow shear area; $M C A=$ middle cerebral artery; OSI = oscillatory shear index; $P C O A=$ posterior communicating artery; PHASES = population, hypertension, age, size of aneurysm, earlier SAH from another aneurysm, site of aneurysm; PLc = pressure loss coefficient; SAH = subarachnoid hemorrhage; VDR = viscous dissipation ratio; WSS = wall shear stress.

SUBMITTED January 23, 2017. ACCEPTED May 8, 2017.

INCLUDE WHEN CITING Published online November 3, 2017; DOI: 10.3171/2017.5.JNS17195. 
Aneurysms are most likely to grow irregularly, ${ }^{1}$ and there is increasing evidence that the rupture itself might significantly alter aneurysm geometry and hemodynamics..$^{14,24,26}$ Therefore, only investigations of aneurysms before rupture can truly answer which factors predict rupture. Reports of up to 13 cases before rupture have emerged, $6,18,24,28$ in which aneurysms were evaluated at various time spans before rupture. None of these are matched on both clinical and aneurysmal factors, and are thus prone to confounding. The aim of this study was to identify hemodynamic factors at the time of diagnosis of unruptured aneurysms that are associated with subsequent rupture.

\section{Methods}

\section{Study Design and Patient Selection}

Norway has a 3-level hierarchical hospital structure. All university clinics (4 centers) with a neurosurgical department treating IA and SAH participated in the study, thus covering the whole population of Norway (5 million people).

The study is a matched case-control study, based on retrospective data collection in the time period from 2003 to 2013. For cases, we identified patients who presented with an unruptured saccular IA that subsequently ruptured. For controls, we identified matching patients with saccular IAs whose aneurysms did not rupture during follow-up.

We recorded the following: date of birth; date of diagnosis for unruptured aneurysm; aneurysm location and parent artery relation (bifurcation/sidewall aneurysm); date admitted with SAH for cases; the reason for the aneurysm being conservatively managed; and known risk factors for $\mathrm{SAH}$ - such as hypertension, ethnicity, smoking status, connective tissue disease, polycystic kidney disease, family history, or prior SAH. We also calculated the PHASES (population, hypertension, age, size of aneurysm, earlier SAH from another aneurysm, site of aneurysm) score. ${ }^{11}$ Patients were excluded if they had undergone previous treatment of the aneurysm of interest, or if the image quality precluded segmentation. Furthermore, cases were excluded if there were multiple aneurysms of which the ruptured one was difficult to identify, and control patients were excluded if they died of an unknown cause during follow-up.

For each case we selected 2 controls who were matched on aneurysm location and size, as well as patient sex and age. The initial matching factor was location-if this factor could not be matched, the patient was excluded. Further matching was done in the order of the factors listed above.

The study was approved by the Northern Norway Regional Committee for Medical Research Ethics, which found the study to be exempt from patient consent. We report the findings according to the STROBE (Strengthening the Reporting of Observational Studies in Epidemiology) guidelines. ${ }^{32}$

\section{Computational Simulation}

We retrieved the first available cerebral angiography study for each patient to perform simulations using the data obtained at the time of diagnosis. Segmentation and meshing of the images was done using the Vascular
Modeling ToolKit (http://www.vmtk.org). All segmented models were inspected by an experienced neurosurgeon to ensure consistency with the original angiography.

We imposed a fully developed pulsatile Womersley flow profile at the model inlets. ${ }^{12}$ At the outlets, we scaled the flow according to the principle of minimal work (Murray's law). In segmented models in which the posterior communicating artery (PCoA) was not merged to the posterior circulation, we assumed zero flux. Walls were assumed to be rigid and impermeable. We assumed blood to behave as a Newtonian fluid. ${ }^{8}$ The Navier-Stokes simulation equations were solved and postprocessed with the open source softwares cbcflow and cbcpost (https:// bitbucket.orgsimula_cbc/), based on the open source finite element library (i.e., FEniCS). ${ }^{19}$ To properly resolve the high-frequency flow dynamics, we resolved the simulations with a temporal resolution of $0.04 \mathrm{msec}$.

The engineering team conducting segmentation and simulation was blinded to the patients' status as case or control, and to any clinical data. The Supplementary Methods provide additional details about patient selection and computational simulation.

\section{Parameter Definitions}

The Supplementary Table specifies abbreviations, mathematical definitions, and pragmatic explanations for each studied parameter. The OSI and LSA are derivatives of WSS. ${ }^{5}$ The PLc, ICI, and VDR are related to the flow phenotype. ${ }^{4,28}$

\section{Statistical Analysis}

Prior to analyses, the distribution of each continuous variable was assessed visually with Q-Q-plots and numerically with the Shapiro-Wilk test. Parametric variables were reported as means with SDs. Nonparametric variables were reported as medians with interquartile range (IQR). Balance between cases and controls was assessed using the Student t-test for parametric variables, and the Wilcoxon rank-sum test for nonparametric variables. Categorical variables were compared using the chi-square test.

Matched case-control analysis was performed using conditional logistic regression, which is appropriate for this study, with 2 controls for each case. ${ }^{22}$ For multivariable regression, backward stepwise elimination of independent variables with a threshold significance level of $>$ 0.2 was used to select the final multivariable model. The data were analyzed with Stata for Mac (version 14, StataCorp LP). A p value of $<0.05$ was assumed to be statistically significant.

\section{Results}

\section{Study Population and Case Matching}

The initial search identified 43 patients with confirmed aneurysmal $\mathrm{SAH}$, originating from saccular aneurysms that were known prior to rupture and had been treated conservatively. Twenty cases were excluded because matching was not accomplished; of these, 18 had aneurysms larger than $10 \mathrm{~mm}$. Eight cases were excluded because of insufficient image quality, and 3 were excluded because of in- 
sufficient image quality in the available controls. Each of the remaining 12 patients were successfully matched to 2 control patients with a confirmed saccular IA that was neither treated nor ruptured during follow-up.

The cases consisted of 4 anterior communicating artery (ACoA; 33.3\%), 7 middle cerebral artery (MCA; 58.3\%), and 1 PCoA $(8.3 \%)$ aneurysm. There were $4(33.3 \%)$ men among cases, and 9 (37.5\%) men among controls $(\mathrm{p}=$ 0.81 ). For cases, the median time from diagnosis to rupture was 3.4 years (IQR 1.6-6.3 years). For controls, the median time from diagnosis to death or end of follow-up was 4.5 years (IQR 3.7-8.2 years). The mean PHASES score was 6.4 (SD 3.0) for cases and 6.5 (SD 2.9) for controls $(p=0.82)$. Table 1 provides baseline characteristics of cases and matched controls.

\section{Hemodynamic Parameters Associated With Future Rupture}

In the univariate analysis, LSA was the only parameter statistically significantly associated with future rupture $(\mathrm{p}$ $=0.041$ ) (Table 2). Minimum logarithmic WSS, averaged logarithmic WSS, PLc, and ICI showed a tendency to be associated with rupture $(\mathrm{p}=0.09,0.14,0.15$, and 0.18 , respectively). Figure 1 shows the distribution of these 5 parameters for cases and controls, and Fig. 2 visualizes WSS and LSA for all case and control aneurysms.

In the multivariable analysis, LSA $(\beta=8.72[95 \% \mathrm{CI}$

TABLE 1. Comparison of baseline characteristics between cases and matched controls

\begin{tabular}{|c|c|c|c|}
\hline Characteristic & $\begin{array}{l}\text { Cases, } \\
n=12\end{array}$ & $\begin{array}{c}\text { Controls, } \\
n=24\end{array}$ & $\begin{array}{c}p \\
\text { Value* }^{*}\end{array}$ \\
\hline \multicolumn{4}{|l|}{ Patients } \\
\hline Male sex & $4(33)$ & $9(38)$ & $0.81 \dagger$ \\
\hline Mean age in yrs & $64.4 \pm 9$ & $63.5 \pm 10$ & $0.80 \dagger$ \\
\hline Current smoker & $6(50)$ & $10(42)$ & 0.64 \\
\hline \multicolumn{4}{|l|}{ Medical Hx } \\
\hline Hypertension & $9(75)$ & $19(79)$ & 0.78 \\
\hline $\begin{array}{l}\text { Family } \mathrm{Hx} \text { of aneurysms } \\
\text { or SAH }\end{array}$ & $1(8)$ & $3(13)$ & 0.71 \\
\hline Previous SAH & $1(8)$ & $0(0)$ & 0.15 \\
\hline Multiple aneurysms & $3(25)$ & $8(33)$ & 0.61 \\
\hline $\begin{array}{l}\text { Treatment of another } \\
\text { aneurysm }\end{array}$ & $1(8)$ & $1(4)$ & 0.61 \\
\hline \multicolumn{4}{|l|}{ Aneurysms } \\
\hline \multicolumn{4}{|l|}{ Location } \\
\hline $\mathrm{ACOA}$ & $4(33)$ & $8(33)$ & \multirow{3}{*}{$1.0 \dagger$} \\
\hline MCA & $7(58)$ & $14(58)$ & \\
\hline PCoA & $1(8)$ & $2(8)$ & \\
\hline Mean max diameter in $\mathrm{mm}$ & $8.34 \pm 3.41$ & $8.15 \pm 3.40$ & $0.87 \dagger$ \\
\hline Mean vol in $\mathrm{mm}^{3}$ & $177.9 \pm 219.4$ & $168.8 \pm 250.8$ & $0.92 \dagger$ \\
\hline Mean PHASES score & $6.4 \pm 3.0$ & $6.5 \pm 2.9$ & 0.82 \\
\hline
\end{tabular}

$\mathrm{Hx}=$ history; $\max =$ maximum

Continuous, parametric variables are reported as the mean \pm SD. Dichotomous and categorical variables are reported as number (\%).

* Statistical significance was set at $p<0.05$.

† Matched variables.
TABLE 2. Univariate analysis of hemodynamic parameters in cases and controls

\begin{tabular}{lccc}
\hline \multirow{2}{*}{ Parameter } & \multicolumn{2}{c}{ Mean Value $( \pm$ SD) } & $p$ \\
\cline { 2 - 3 } & \multicolumn{1}{c}{ Cases } & Controls & Value \\
\hline Min WSS & $0.62(0.66)$ & $0.84(0.69)$ & 0.39 \\
\hline Time- \& space-avg WSS & $6.33(4.70)$ & $7.50(3.42)$ & 0.41 \\
\hline Max WSS & $39.6(23.5)$ & $55.2(54.1)$ & 0.39 \\
\hline Min logarithmic WSS & $-1.17(1.39)$ & $-0.47(0.79)$ & 0.09 \\
\hline Avg logarithmic WSS & $0.96(1.01)$ & $1.37(0.53)$ & 0.14 \\
\hline OSI & $0.046(0.039)$ & $0.039(0.025)$ & 0.47 \\
\hline LSA & $0.15(0.16)$ & $0.06(0.07)$ & 0.041 \\
\hline PLC & $1.25(0.31)$ & $1.48(0.45)$ & 0.15 \\
\hline ICI & $2.28(1.12)$ & $1.90(1.17)$ & 0.18 \\
\hline SCI & $4.07(2.38)$ & $4.31(2.44)$ & 0.76 \\
\hline VDR & $0.31(0.23)$ & $0.46(0.63)$ & 0.49 \\
\hline Avg = avan
\end{tabular}

$A v g=$ averaged $; \min =$ minimum; $\mathrm{SCl}=$ shear concentration index.

Boldface type indicates statistical significance $(p<0.05)$.

$0.85-16.59], \mathrm{p}=0.030)$ and ICI $(\beta=1.13$ [95\% CI -0.30 to $2.55], \mathrm{p}=0.12$ ) were retained in the conditional logistic regression model. Their $\mathrm{p}$ values remained almost constant when adjusting for the matching variables patient sex, age, aneurysm location and size, as well as smoking status, previous SAH, hypertension, and PHASES score (data not shown).

\section{Discussion}

This study indicates that, already at the time of diagnosis, unruptured aneurysms that later ruptured had statistically significantly higher LSAs than aneurysms that did not rupture. To our knowledge, this study is one of very few to investigate hemodynamics in prerupture aneurysms, and the first that matches cases and controls on both patient and aneurysmal characteristics.

\section{Isolating Hemodynamic Properties}

Unmatched studies reporting WSS differences between ruptured and unruptured aneurysms might simply observe the effect of other factors on hemodynamics. Lauric et al. observed a strong negative correlation between WSS and aneurysm volume $\left(\mathrm{R}^{2}=0.97\right) .{ }^{16}$ The importance of matching based on clinical factors is illustrated in a prospective study that estimated the joint hazard ratio for aneurysm rupture associated with smoking and hypertension to be 13.3 (95\% CI 6.9-25.4). ${ }^{17}$

Future tools to aid patient-specific treatment choices must probably encompass both clinical and aneurysmal factors to increase predictive power. ${ }^{7}$ However, to understand the contribution of hemodynamics to the overall rupture risk, a matched study reducing possible confounding from aneurysm size and location, and patient sex and age is essential.

\section{Prerupture Hemodynamics}

Our study shows that larger areas of low WSS and a concentrated inflow jet might constitute some of the ear- 

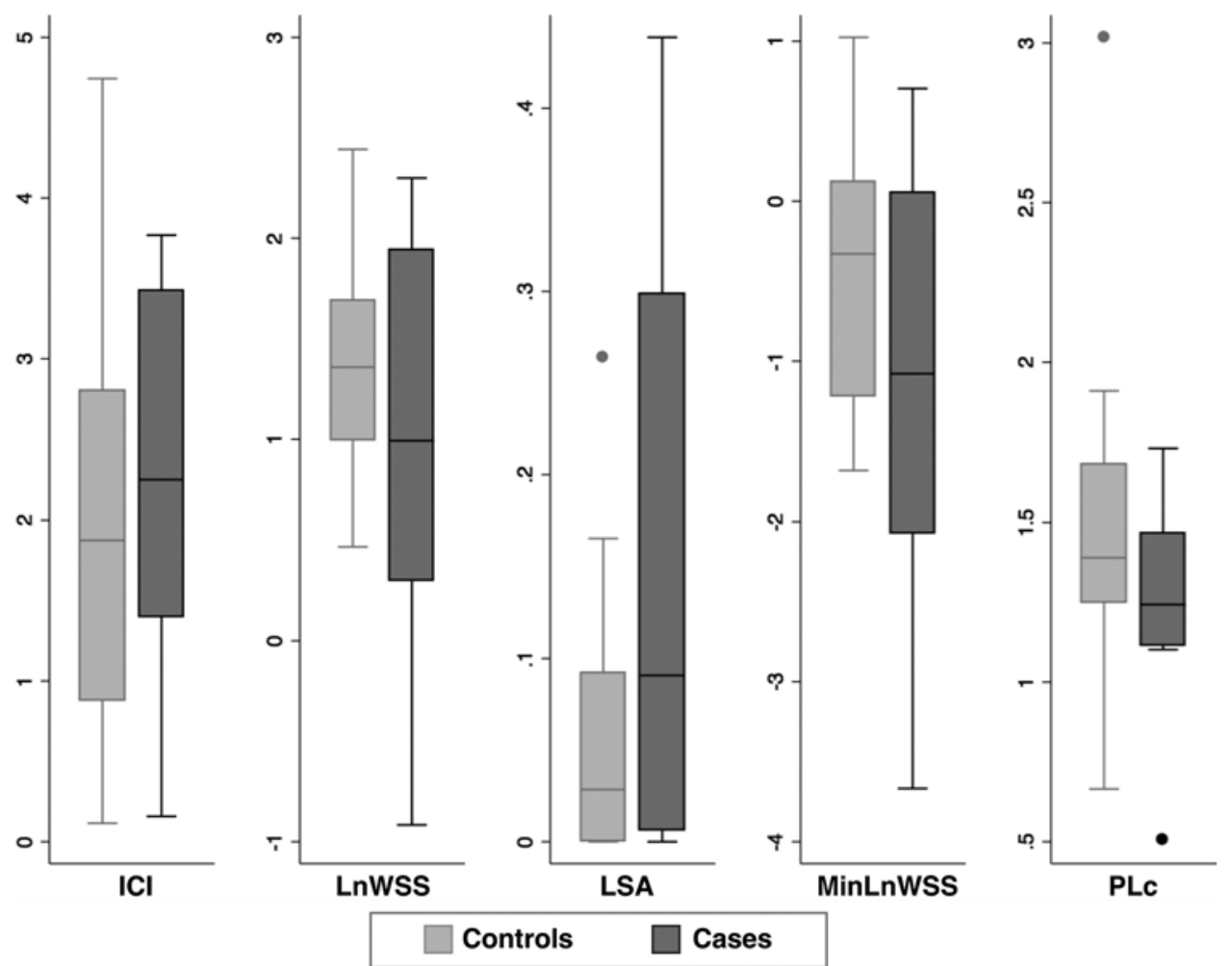

FIG. 1. Boxplot showing graphic representation of the distribution of selected hemodynamic parameters in cases and controls. LnWSS = averaged logarithmic WSS; MinLnWSS = minimum logarithmic WSS.

liest detectable hemodynamic patterns in an aneurysm's natural history from formation to rupture.

The friction between blood and endothelial cells induces mechanobiological signaling essential for normal arterial wall proliferation. Low or stagnant flow causes an inflammatory response in the vascular wall, promoting atherogenesis and leukocyte infiltration. ${ }^{20}$ In histological analyses of ruptured and unruptured aneurysms, Frösen et al. found that ruptured aneurysms were characterized by inflammatory infiltration as well as apoptosis and degeneration of the wall matrix. ${ }^{10}$ However, alterations in blood-endothelial signaling cannot serve as a complete explanation. Frösen et al. also found that $62 \%$ of ruptured aneurysms lacked endothelium, compared with $30 \%$ of unruptured aneurysms. In addition to shear stress, cyclic pressure induces stress in the vascular wall sensed by the deeper smooth muscle. In computational fluid dynamics (CFD) studies, this mechanotransduction is not subject to study because walls must be assumed rigid. Other methods, such as fluid structure interaction analyses, can investigate forces other than shear stress, because they are not limited to the rigid wall assumption. However, fluid structure interaction analysis introduces additional assumptions into the model, and has higher computational resource requirements.

Our findings are in accordance with other studies indicating that hemodynamic parameters might predict rupture. Takao et al. found significantly lower PLc among 13 internal carotid artery (ICA) and MCA aneurysms that later ruptured, compared with 87 control aneurysms. The 7 ICA aneurysms in the study also had lower minimum
WSS. ${ }^{28}$ However, the authors did not report patient characteristics, and although the aneurysms belonged to the same size category, the mean size was larger for aneurysms that later ruptured than for those that did not. Duan et al. ${ }^{6}$ matched 6 patients with PCoA aneurysms that later ruptured to 4 controls each, based on clinical factors. The aneurysms that later ruptured showed larger LSA and lower normalized WSS. Case and control aneurysms were not matched for size, and the mean size was significantly larger for the aneurysms that later ruptured (mean $7.8 \mathrm{~mm}$ ) than for those that did not (mean $4.7 \mathrm{~mm}$ ). ${ }^{6}$

Aneurysm growth and aneurysm rupture are considered to be closely related. Importantly, Brinjikji et al. also found statistically significantly larger LSA in 12 aneurysms that grew for at least 1 year, compared with 12 matched, stable aneurysms. ${ }^{2}$

\section{Limitations of the Study}

Because all patients in this study were treated conservatively, they can be assumed to be older or to have more comorbidities than the general population of patients with aneurysms, or the aneurysms may have seemed more benign to the responsible clinicians. Matching can further decrease generalizability, but increases internal validity and statistical power. ${ }^{9}$ Of note, the matching caused large aneurysms to be underrepresented, because patients with such aneurysms are quickly treated and not readily available as controls. However, this selection bias caused a relative emphasis on small- and moderate-sized aneurysms, in which decision support is needed the most. We also be- 
MCA CASE

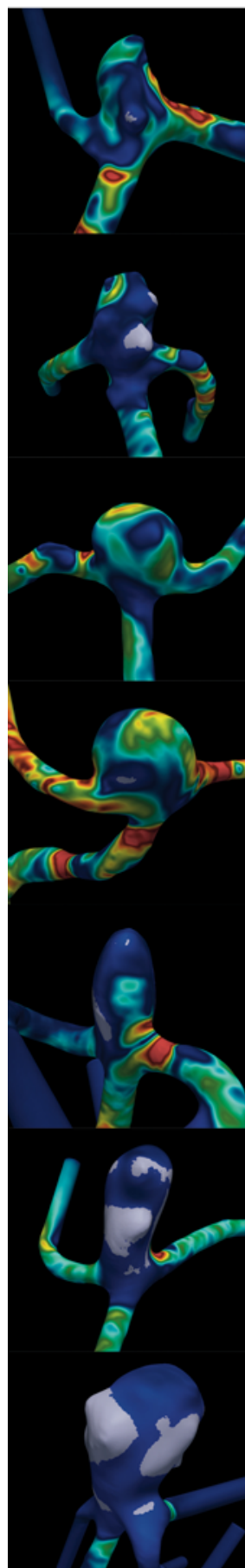

CONTROL 1

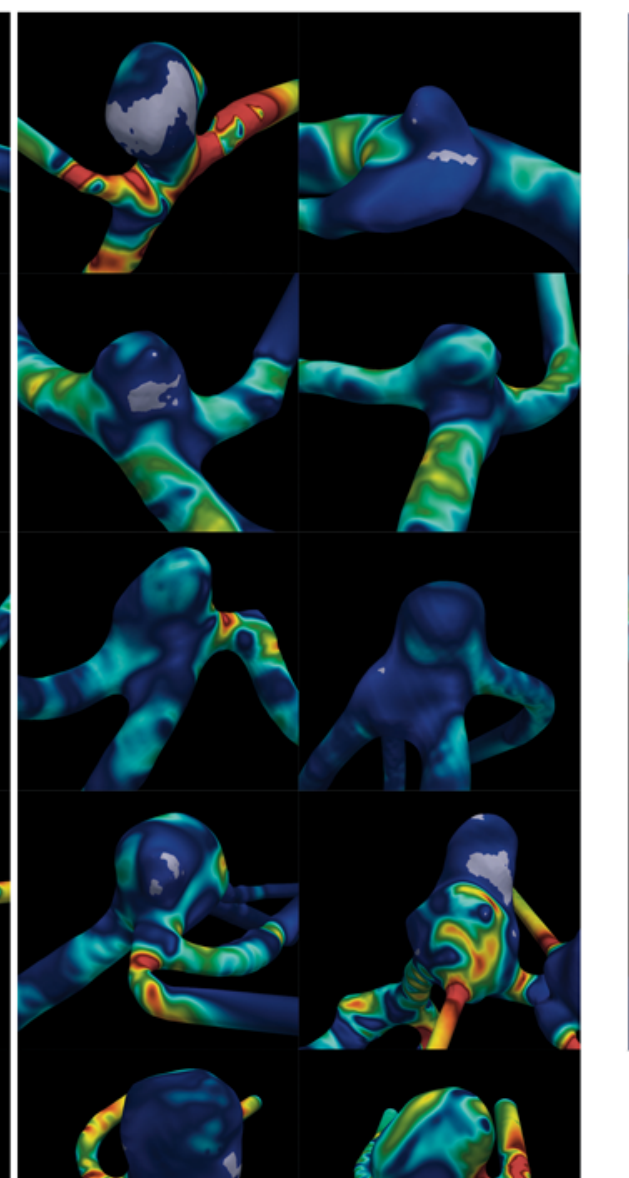

ACOM

CASE

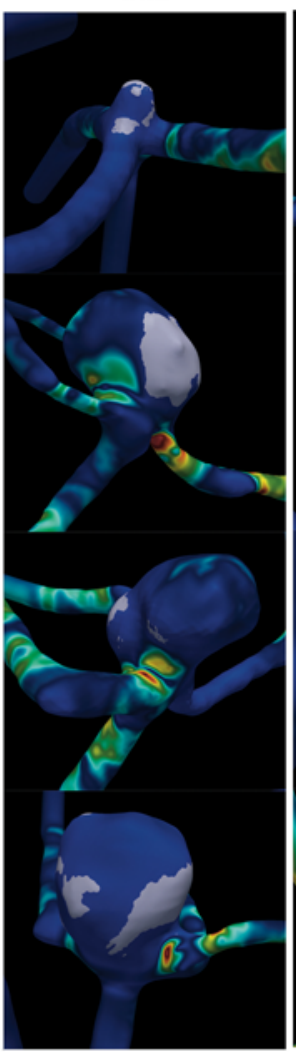

PCOM
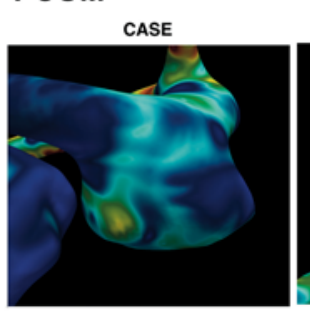

CONTROL 1

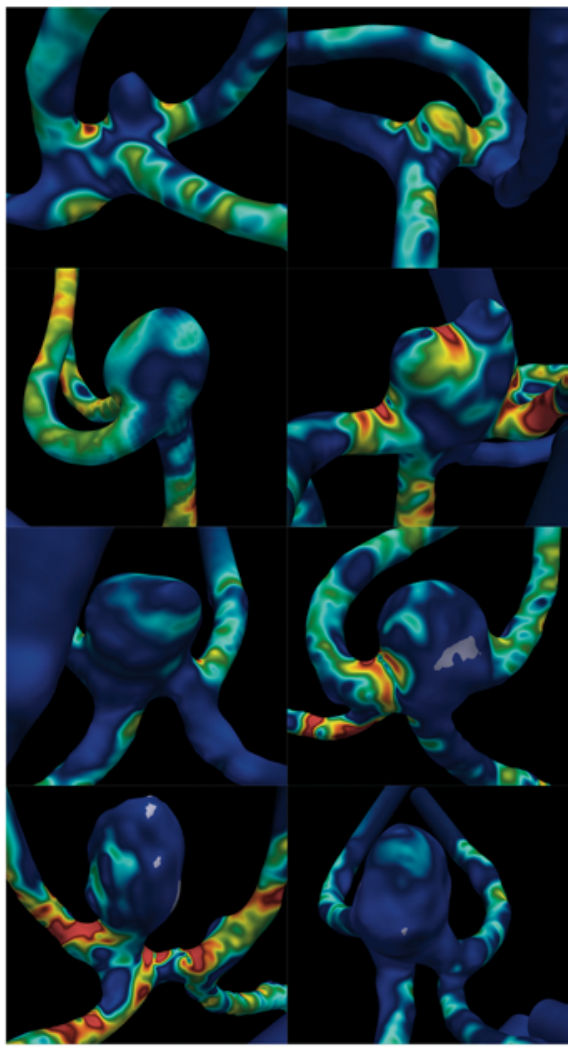

CONTROL 2

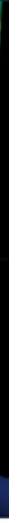

FIG. 2. Visualization of WSS and LSA for all case and control aneurysms, grouped by location. The WSS is represented by a color scale ranging from blue for WSS $<1.0 \mathrm{~Pa}$, to red for WSS $>10 \mathrm{~Pa}$. Areas of low shear are colored gray, and represent areas of the dome where the average WSS is below $10 \%$ of the average WSS in the parent artery. ACOM = ACOA; PCOM $=P C O A$.

lieve that the matching design is highly warranted to control for known confounders of hemodynamic parameters.

More study patients are needed to allow for definite conclusions. Conservatively managed aneurysms that are followed until rupture or with a long follow-up time without rupture are rare, but the statistical power of the present study is increased because we managed to match 2 controls to each case. Whether any of the control aneurysms would eventually rupture during further follow-up is unknown. If that were the case, the findings of this study suffer from a dilution bias in which effect sizes are larger in a general group of patients with aneurysms than in our study 
sample. None of the clinical risk factors such as previous $\mathrm{SAH}$, multiple aneurysms, and smoking status were statistically significantly different between cases and controls. The retrospective nature of the study reduces the accuracy of the patient risk factors recorded, and we cannot rule out any selection bias from these factors.

Previous CFD studies have revealed partially conflicting results. ${ }^{14}$ The segmentation process introduces uncertainty, and the computational simulation depends on assumptions of given boundary conditions, fixed walls, and rheology model, as well as the numerical methods used. ${ }^{29}$ Still, CFD is increasingly used within the field of biomechanics, and adds to a growing knowledge about the role and importance of hemodynamic forces in vascular pathophysiology.

Follow-up of conservatively managed aneurysms is still required, because our findings are insufficient for a conclusive rupture prediction at the time of diagnosis. Future studies investigating the hemodynamic trends at several points from diagnosis to rupture can shed light onto which hemodynamic changes are causal to later hemodynamic differences. Furthermore, improved simulation methods incorporating more patient-specific input variables might minimize simulation setup uncertainties. Despite limitations, however, this study presents long-awaited data on the association between prerupture hemodynamics and future rupture risk.

\section{Conclusions}

The hemodynamics of unruptured aneurysms at the time of diagnosis are different in aneurysms that later rupture than in those that remain unruptured, even with rigorously matched case and control aneurysms. Increased LSA might be an early predictor of rupture.

\section{Acknowledgments}

Northern Norway Regional Health Authority and UiT The Arctic University of Norway funded the study. The funders had no role in the study design, data collection, data analysis, data interpretation, writing of the manuscript, or decision to submit for publication.

We thank Haakon Lindekleiv (UiT The Arctic University of Norway and University Hospital of Northern Norway); Angelika and Wilhelm Sorteberg (Rikshospitalet, Oslo, Norway); Ole Solheim (St. Olav Hospital, Trondheim, Norway); and Rune Grov Eilertsen (University Hospital of Northern Norway) for participating in the data collection. Also, we thank Kent-Andre Mardal and Kristian Valen-Sendstad (Simula Research Laboratory, Oslo, Norway) for valuable discussions and suggestions. The simulations performed in this study were performed on the Abel Cluster (project NN9279K), owned by the University of Oslo and the Norwegian metacenter for High Performance Computing (NOTUR), and operated by the Department for Research Computing at USIT, the University of Oslo IT department (http://www.hpc.uio.no/).

\section{References}

1. Bor AS, Tiel Groenestege AT, terBrugge KG, Agid R, Velthuis BK, Rinkel GJ, et al: Clinical, radiological, and flow-related risk factors for growth of untreated, unruptured intracranial aneurysms. Stroke 46:42-48, 2015

2. Brinjikji W, Chung BJ, Jimenez C, Putman C, Kallmes DF, Cebral JR: Hemodynamic differences between unstable and stable unruptured aneurysms independent of size and location: a pilot study. J Neurointerv Surg 9:376-380, 2017

3. Can A, Du R: Association of hemodynamic factors with intracranial aneurysm formation and rupture: systematic review and meta-analysis. Neurosurgery 78:510-520, 2016

4. Cebral JR, Castro MA, Burgess JE, Pergolizzi RS, Sheridan MJ, Putman CM: Characterization of cerebral aneurysms for assessing risk of rupture by using patient-specific computational hemodynamics models. AJNR Am J Neuroradiol 26:2550-2559, 2005

5. Chien S: Mechanotransduction and endothelial cell homeostasis: the wisdom of the cell. Am J Physiol Heart Circ Physiol 292:H1209-H1224, 2007

6. Duan G, Lv N, Yin J, Xu J, Hong B, Xu Y, et al: Morphological and hemodynamic analysis of posterior communicating artery aneurysms prone to rupture: a matched case-control study. J Neurointerv Surg 8:47-51, 2016

7. Etminan N, Rinkel GJ: Unruptured intracranial aneurysms: development, rupture and preventive management. Nat Rev Neurol 12:699-713, 2016

8. Evju $\varnothing$, Valen-Sendstad K, Mardal KA: A study of wall shear stress in 12 aneurysms with respect to different viscosity models and flow conditions. J Biomech 46:2802-2808, 2013

9. Freemantle N, Marston L, Walters K, Wood J, Reynolds MR, Petersen I: Making inferences on treatment effects from real world data: propensity scores, confounding by indication, and other perils for the unwary in observational research. BMJ 347:f6409, 2013

10. Frösen J, Piippo A, Paetau A, Kangasniemi M, Niemelä M, Hernesniemi J, et al: Remodeling of saccular cerebral artery aneurysm wall is associated with rupture: histological analysis of 24 unruptured and 42 ruptured cases. Stroke 35:22872293, 2004

11. Greving JP, Wermer MJ, Brown RD Jr, Morita A, Juvela S, Yonekura M, et al: Development of the PHASES score for prediction of risk of rupture of intracranial aneurysms: a pooled analysis of six prospective cohort studies. Lancet Neurol 13:59-66, 2014

12. Hoi Y, Wasserman BA, Xie YJ, Najjar SS, Ferruci L, Lakatta EG, et al: Characterization of volumetric flow rate waveforms at the carotid bifurcations of older adults. Physiol Meas 31:291-302, 2010

13. Johnston SC, Selvin S, Gress DR: The burden, trends, and demographics of mortality from subarachnoid hemorrhage. Neurology 50:1413-1418, 1998

14. Kallmes DF: Point: CFD-computational fluid dynamics or confounding factor dissemination. AJNR Am J Neuroradiol 33:395-396, 2012

15. Kono K, Tomura N, Yoshimura R, Terada T: Changes in wall shear stress magnitude after aneurysm rupture. Acta Neurochir (Wien) 155:1559-1563, 2013

16. Lauric A, Hippelheuser J, Cohen AD, Kadasi LM, Malek AM: Wall shear stress association with rupture status in volume matched sidewall aneurysms. J Neurointerv Surg 6:466-473, 2014

17. Lindekleiv H, Sandvei MS, Romundstad PR, Wilsgaard T, Njølstad I, Ingebrigtsen T, et al: Joint effect of modifiable risk factors on the risk of aneurysmal subarachnoid hemorrhage: a cohort study. Stroke 43:1885-1889, 2012

18. Liu J, Fan J, Xiang J, Zhang Y, Yang X: Hemodynamic characteristics of large unruptured internal carotid artery aneurysms prior to rupture: a case control study. J Neurointerv Surg 8:367-372, 2016

19. Logg A, Mardal KA, Wells GN (eds): Automated Solution of Differential Equations by the Finite Element Method. Berlin: Springer, 2012

20. Meng H, Tutino VM, Xiang J, Siddiqui A: High WSS or low WSS? Complex interactions of hemodynamics with intracranial aneurysm initiation, growth, and rupture: toward a uni- 
fying hypothesis. AJNR Am J Neuroradiol 35:1254-1262, 2014

21. Müller TB, Sandvei MS, Kvistad KA, Rydland J, Håberg A, Vik A, et al: Unruptured intracranial aneurysms in the Norwegian Nord-Trøndelag Health Study (HUNT): risk of rupture calculated from data in a population-based cohort study. Neurosurgery 73:256-261, 2013

22. Niven DJ, Berthiaume LR, Fick GH, Laupland KB: Matched case-control studies: a review of reported statistical methodology. Clin Epidemiol 4:99-110, 2012

23. Pereira VM, Brina O, Bijlenga P, Bouillot $P$, Narata AP, Schaller K, et al: Wall shear stress distribution of small aneurysms prone to rupture: a case-control study. Stroke 45:261-264, 2014

24. Rahman M, Ogilvy CS, Zipfel GJ, Derdeyn CP, Siddiqui AH, Bulsara KR, et al: Unruptured cerebral aneurysms do not shrink when they rupture: multicenter collaborative aneurysm study group. Neurosurgery 68:155-161, 2011

25. Rivero-Arias O, Gray A, Wolstenholme J: Burden of disease and costs of aneurysmal subarachnoid haemorrhage (aSAH) in the United Kingdom. Cost Eff Resour Alloc 8:6, 2010

26. Schneiders JJ, Marquering HA, van den Berg R, VanBavel E, Velthuis B, Rinkel GJ, et al: Rupture-associated changes of cerebral aneurysm geometry: high-resolution 3D imaging before and after rupture. AJNR Am J Neuroradiol 35:13581362,2014

27. Steiner T, Juvela S, Unterberg A, Jung C, Forsting M, Rinkel G: European Stroke Organization guidelines for the management of intracranial aneurysms and subarachnoid haemorrhage. Cerebrovasc Dis 35:93-112, 2013

28. Takao H, Murayama Y, Otsuka S, Qian Y, Mohamed A, Masuda S, et al: Hemodynamic differences between unruptured and ruptured intracranial aneurysms during observation. Stroke 43:1436-1439, 2012

29. Valen-Sendstad K, Steinman DA: Mind the gap: impact of computational fluid dynamics solution strategy on prediction of intracranial aneurysm hemodynamics and rupture status indicators. AJNR Am J Neuroradiol 35:536-543, 2014

30. van Gijn J, Rinkel GJ: Subarachnoid haemorrhage: diagnosis, causes and management. Brain 124:249-278, 2001

31. Vlak MH, Algra A, Brandenburg R, Rinkel GJ: Prevalence of unruptured intracranial aneurysms, with emphasis on sex, age, comorbidity, country, and time period: a systematic review and meta-analysis. Lancet Neurol 10:626-636, 2011

32. von Elm E, Altman DG, Egger M, Pocock SJ, Gøtzsche PC, Vandenbroucke JP: The Strengthening the Reporting of Observational Studies in Epidemiology (STROBE) statement: guidelines for reporting observational studies. Ann Intern Med 147:573-577, 2007

\section{Disclosures}

The authors report no conflict of interest concerning the materials or methods used in this study or the findings specified in this paper.

\section{Author Contributions}

Conception and design: Skodvin, Isaksen. Acquisition of data: all authors. Analysis and interpretation of data: all authors. Drafting the article: all authors. Critically revising the article: all authors. Reviewed submitted version of manuscript: all authors. Approved the final version of the manuscript on behalf of all authors: Skodvin. Statistical analysis: Skodvin. Administrative/technical/ material support: all authors. Study supervision: Skodvin, Isaksen.

\section{Supplemental Information}

Online-Only Content

Supplemental material is available with the online version of the article.

Supplementary Methods and Table. https://thejns.org/doi/ suppl/10.3171/2017.5.JNS17195.

\section{Previous Presentations}

Portions of this work were presented in abstract form at the Norwegian Neurosurgery Association Annual Meeting in Oslo, Norway, on October 27, 2016.

\section{Correspondence}

Torbjørn Øygard Skodvin, Varden 197, Tromsø 9018, Norway. email: torbjorn.skodvin@uit.no. 